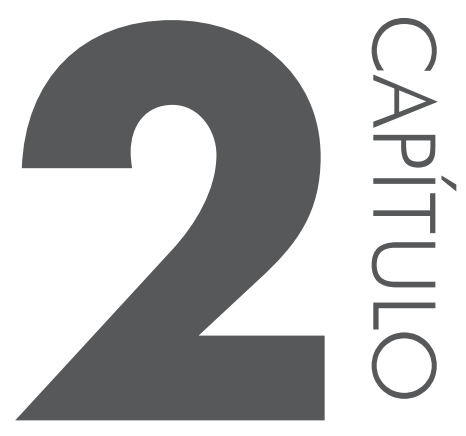

\title{
MECÂNICA DO CONTATO
}

\subsection{A TEORIA LINEAR ELÁSTICA DE HERTZ}

Segundo Johnson (1987), a primeira abordagem do estudo de contato entre corpos elásticos homogêneos foi feita por Heinrich Hertz, aos 24 anos, em 1882, na Universidade de Berlim, no clássico artigo "On the contact of elastic solids". A questão surgiu quando a deformação elástica das lentes, objeto de seus estudos, causavam influências nos padrões das franjas de interferência devido às forças de contato na fixação delas.

O conhecimento de Hertz sobre a Teoria do Potencial Eletrostático serviu para que se demonstrasse, por analogia, que uma distribuição semielipsoidal de pressões de contato produziriam deslocamentos elásticos em ambos os corpos, compatíveis com a proposta de uma área de contato elíptica, para o caso mais geral de contato entre os corpos.

A teoria de Hertz está restrita a superfícies sem atrito e a sólidos perfeitamente elásticos. O progresso na mecânica do contato, posteriormente a Hertz, se deu no sentido de se remover tais restrições.

Ainda de acordo com a teoria hertziana, pode-se confinar a atenção àquela parte de cada corpo muito próxima do contato, já que, ali, as tensões são muito maiores que as que ocorrem em qualquer outra parte. Além disso, as dimensões 
da região do contato são, geralmente, muito pequenas se comparadas ao tamanho dos corpos. O mesmo não se pode dizer de corpos que se conformam entre si, uma vez que a área da superfície de contato das superfícies conformadas não é desprezível se comparada às demais dimensões dos corpos em questão.

A transferência de esforços mecânicos entre dois corpos ocorre por meio do contato entre superfícies. No caso em que pelo menos uma dessas superfícies é curva, os esforços resultantes na região de contato são conhecidos como tensões de contato. Para prevenir a possibilidade de falha da superfície, nesses casos, é necessário calcular e compreender os estados de tensão que resultam do carregamento de um corpo sobre o outro.

Neste trabalho, o foco se restringirá aos aspectos da mecânica do contato entre superfícies em não conformação, isto é, a problemas de contato não conforme. O diagrama da Figura 2.1 representa a geometria do contato no problema a ser estudado aqui. Hertz, entretanto, não avançou até este caso do plano revestido.

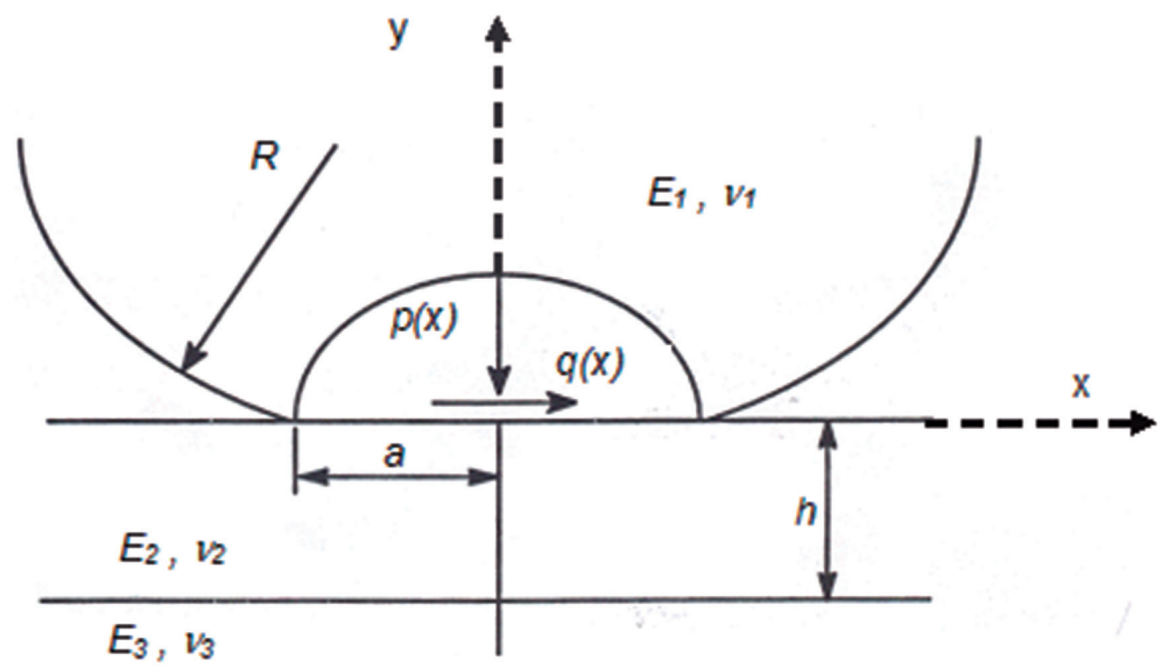

Figura 2.1 Região de contato entre cilindro (1) e semiplano infinito (3) com revestimento (2), com distribuição de pressão semielipsoidal (segundo Hertz).

Para a configuração do estado de tensão, selecionou-se a origem de um sistema de coordenadas no centro da área de contato, com o eixo " $x$ " perpendicular ao plano formado pelo eixo do cilindro e a linha de contato inicial, o eixo " $y$ " no plano da força de contato e o eixo "z" paralelo ao eixo do cilindro, conforme Figura 2.1.

As simplificações propostas por Hertz para o seu modelo foram as seguintes:

- as superfícies são contínuas e em não conformação;

- as deformações na região de contato são muito pequenas; 
- cada sólido pode ser considerado como um semiespaço elástico, onde a semilargura de contato é muito menor que o raio de curvatura de cada corpo, bem como de ambas as dimensões laterais e de profundidade dos corpos em contato;

- ausência de atrito entre as superfícies.

Se as superfícies em contato são, por exemplo, duas esferas, a área de contato obtida é circular de raio $a$. A pressão em cada esfera tem uma distribuição semielíptica e a pressão máxima, $\mathrm{P}_{0}$, ocorre no centro da área de contato. No entanto, quando as superfícies em contato são cilíndricas de eixos paralelos, a área de contato é um retângulo estreito, de largura, $2 a$, onde:

onde:

$$
\tau_{1}=\sqrt{\frac{2 F d^{*}}{L E^{*}}}
$$

$$
\begin{gathered}
\frac{1}{E^{*}}=\frac{1-\boldsymbol{V}_{1}^{2}}{E_{1}}+\frac{1-\boldsymbol{v}_{2}^{2}}{E_{2}} \\
\frac{1}{d^{*}}=\frac{1}{d_{1}}+\frac{1}{d_{2}}
\end{gathered}
$$

sendo que:

- L é o comprimento da área de contato,

- F é a força aplicada,

- $v_{1}$ e $v_{2}$ é o coeficiente de Poisson,

- $d^{*}, d_{1}$ e $d_{2}$ são os diâmetros das respectivas superfícies cilíndricas, quando ambas são consideradas flexíveis.

- $R$ é o raio do indentador cilíndrico.

A pressão, nesse caso, tem uma distribuição elíptica segundo a largura 2a e a pressão máxima $\left(\mathrm{P}_{0}\right)$ é dada por:

$$
\mathrm{P}_{0}=\frac{2 F}{\pi b l}
$$

As tensões principais obtidas pela integração ao longo do eixo $y$ são as seguintes, segundo Johnson (1987):

$$
\begin{aligned}
& \sigma_{x}=-\frac{p_{0}}{a}\left\{\left(a^{2}+2 y^{2}\right)\left(a^{2}+y^{2}\right)^{-0.5}-2 y\right\} \\
& \sigma_{y}=-\frac{p_{0}}{a}\left(a^{2}+y^{2}\right)^{-0.5} \\
& \sigma_{z}=v\left(\sigma_{x}+\sigma_{y}\right)
\end{aligned}
$$




$$
\tau_{1}=-\frac{p_{0}}{a}\left\{y-y^{2}\left(a^{2}+y^{2}\right)^{-0.5}\right\}
$$

sendo que em $y=-(0.78$ a) ocorre

$$
\left(\tau_{1}\right)_{\max }=0.304 \mathrm{P}_{0}
$$

As equações (2.2) e (2.3) podem ser aplicadas a um cilindro e a uma superfície plana, fazendo-se $d_{2}=\infty$ para o plano. Vale ressaltar que as equações (2.5), (2.6) e (2.7) independem do coeficiente de Poisson para estado plano de deformação $(E P D)$, exceto $\sigma_{\mathrm{z}}$. Estando determinados a $\mathrm{P}_{0}$ e o semicontato, $a$, as tensões em qualquer ponto do eixo $y$ de aplicação da carga podem ser calculadas, segundo as equações anteriores, que estão plotadas no gráfico da Figura 2.2, segundo Johnson (1987):

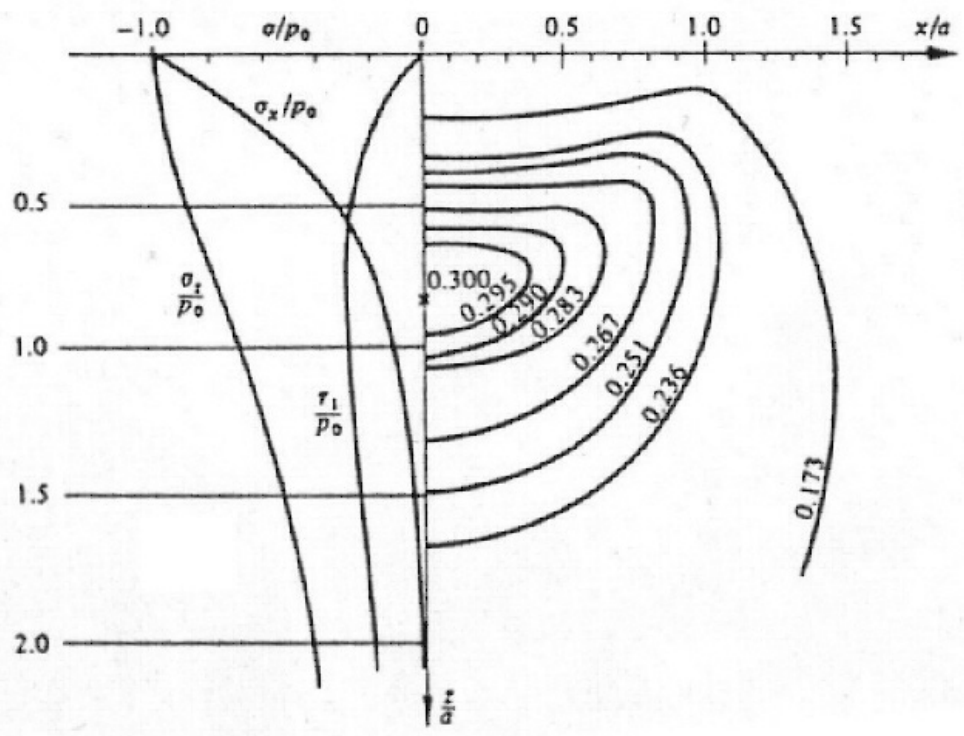

(a)

Figura 2.2 Contato entre dois cilindros. (a) Cálculo das tensões ao longo do eixo de aplicação da carga normal, segundo Johnson (1987), (b) Contorno da primeira tensão cisalhante principal $\tau_{1} .0$ referencial (z/a) adotado pela referência anterior equivale a $(-y / a)$ no sistema de coordenadas aqui adotado.

\subsection{CARREGAMENTO NORMAL E TANGENCIAL}

Em uma discussão preliminar, é importante que se façam distinguir dois conceitos importantes para este problema: 
- movimento deslizante: consiste de uma velocidade periférica relativa entre as superfícies nos seus pontos de contato;

- movimento com rolamento: envolve uma velocidade angular relativa entre dois corpos com respeito aos eixos paralelos ao plano tangente a essas superfícies.

Uma força normal $P$ pressionando dois corpos entre si dá origem a uma área de contato que, na ausência de forças de fricção, teria as dimensões dadas pela teoria de Hertz. Entretanto, até mesmo uma tendência ao deslizamento introduz uma força tangencial $Q$, que atua em cada uma das superfícies em contato, em um sentido que se opõe ao seu movimento.

O caso a ser investigado é aquele em que os corpos estão sujeitos a uma força tangencial que tende a levá-los ao deslizamento. Para esse fim, recorre-se à premissa básica de Hertz que diz que dois corpos podem ser considerados como um semiespaço elástico nas proximidades do contato.

A componente $\bar{u}_{y}$, devido a $Q$ (ver Figura 2.3), é proporcional à constante elástica $\frac{1-2 v}{G}$. Tendo esforços tangenciais, que agem na interface das superfícies em contato de ambos os corpos, e magnitudes iguais, em sentidos opostos, então, segundo Johnson (1987), tem-se que:

$$
q_{1}(x, y)=-q_{2}(x, y) \Leftrightarrow \frac{G_{1}}{1-2 v_{1}} \bar{u}_{z_{1}}(x, y)=-\frac{G_{2}}{1-2 v_{2}} \bar{u}_{z_{2}}(x, y)
$$

$\mathrm{Na}$ análise do problema de contato envolvendo esforços tangenciais, a pressão normal e a tensão tangencial serão assumidas como independentes uma da outra, sendo possível, assim, a superposição dos resultados para encontrar o campo de tensões resultantes.

Uma pressão normal $p(x)$ e um tensão tangencial $q(x)$ estão distribuídas arbitrariamente no intervalo $(-b<x<a)$, em um semiespaço elástico, conforme mostra a Figura 2.3. Desejam-se encontrar as componentes de tensões devidas a $p(x)$ e $q(x)$ em um ponto $A$ qualquer do plano e o deslocamento de um ponto $C$ qualquer na superfície do contato.

As trações que atuam no ponto $B$ da superfície, distante $s$ de $O$, em um elemento de área de largura $d s$, podem ser consideradas como forças concentradas de magnitude $p . d s$, atuando perpendicularmente à superfície, e $q . d s$, tangencialmente à superfície. 


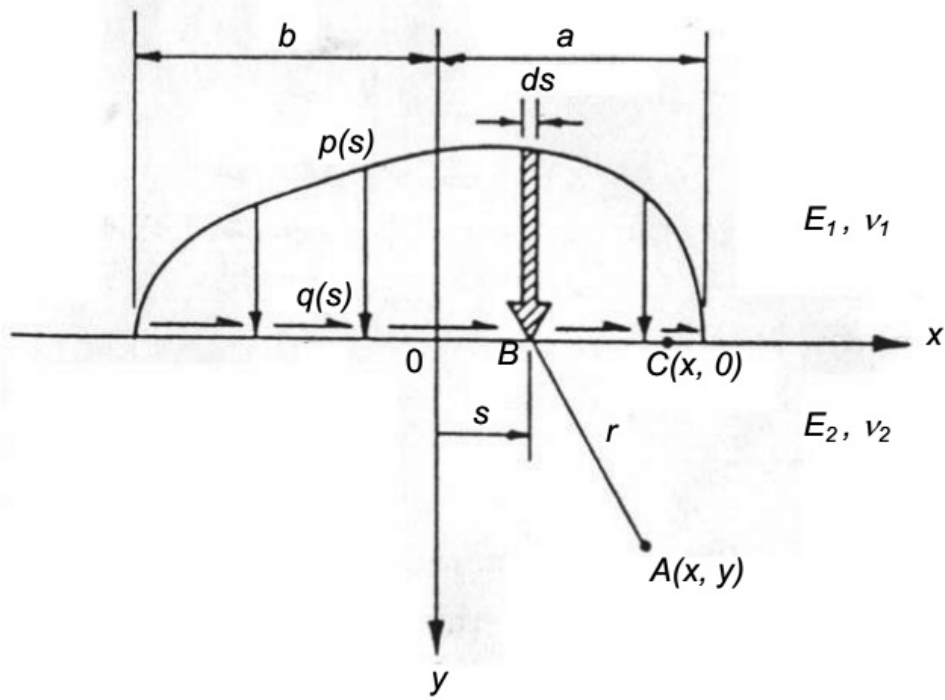

Figura 2.3 Tensões devidas às distribuições tangencial e normal, conforme Johnson (1987), cujo referencial adotado em y equivale $a-y$ neste trabalho.

Da integração dessas forças em todo o intervalo $(-b<x<a)$, resultam as componentes de tensão em $A$, devidas à distribuição completa de $\mathrm{p}(\mathrm{x})$ e $\mathrm{q}(\mathrm{x})$, segundo Johnson (1987):

$$
\begin{aligned}
& \sigma_{x}=\frac{-2 z}{\pi} \int_{-b}^{a} \frac{p(s)(x-s)^{2} d s}{\left\{(x-s)^{2}+z^{2}\right\}^{2}}-\frac{2}{\pi} \int_{-b}^{a} \frac{q(s)(x-s)^{3} d s}{\left\{(x-s)^{2}+z^{2}\right\}^{2}} \\
& \sigma_{z}=\frac{-2 z^{3}}{\pi} \int_{-b}^{a} \frac{p(s) d s}{\left\{(x-s)^{2}+z^{2}\right\}^{2}}-\frac{2 z^{2}}{\pi} \int_{-b}^{a} \frac{q(s)(x-s) d s}{\left\{(x-s)^{2}+z^{2}\right\}^{2}} \\
& \tau_{x z}=\frac{-2 z^{2}}{\pi} \int_{-b}^{a} \frac{p(s)(x-s) d s}{\left\{(x-s)^{2}+z^{2}\right\}^{2}}-\frac{2 z}{\pi} \int_{-b}^{a} \frac{q(s)(x-s)^{2}}{\left\{(x-s)^{2}+z^{2}\right\}^{2}}
\end{aligned}
$$

Nos casos em que as constantes elásticas dos dois corpos em contato forem iguais, a distribuição das trações tangenciais de um semiespaço elástico, em estado plano de deformação (EPD), para o caso sem deslizamento, é dada por:

$$
q(x)=\frac{Q}{\sqrt{\pi\left(a^{2}-x^{2}\right)}}
$$


Para problemas com constantes elásticas diferentes, pode-se lançar mão do estudo da influência de uma diferença nas constantes elásticas feito por Bufler (1959), em que as tensões superficiais na área de contato são dadas por:

$$
q(x)=\frac{\mu E^{*}}{2 R\left(1+\beta^{2} \mu^{2}\right)^{\frac{1}{2}}}\left(\frac{a+x}{a-x}\right)^{\gamma}\left(a^{2}-x^{2}\right)^{\frac{1}{2}}
$$

onde:

$$
\begin{gathered}
\beta=\frac{1}{2}\left[\frac{\left\{\left(1-2 v_{1}\right) / G_{1}\right\}-\left\{\left(1-2 v_{2}\right) / G_{2}\right\}}{\left\{\left(1-v_{1}\right) / G_{1}\right\}+\left\{\left(1-v_{2}\right) / G_{2}\right\}}\right] \\
\text { e } \gamma \cong-\beta \mu / \pi .
\end{gathered}
$$

A semilargura de contato é dada por:

$$
a^{2}=\frac{1}{1-4 \gamma^{2}} \frac{4 P R}{E^{*}}
$$

Se $\beta=0$, as constantes dos dois corpos são iguais e $\gamma$ desaparece. Nesse caso, a área de contato passa a ser dada pela teoria Hertziana.

\subsection{TENDÊNCIA AO DESLIZAMENTO ENTRE CORPOS ELÁSTICOS}

Se a força tangencial não for suficiente para extrapolar o valor limite da força de atrito e se não houver deslizamento relativo entre os corpos em contato, então o problema que surge devido à combinação das forças normal e tangencial se enquadra no caso em estudo.

A força normal P origina uma área de contato e uma distribuição de pressão que, como já dito, são assumidas como sendo independentes da força tangencial Q. Portanto, é dada pela teoria de Hertz. O efeito da força Q é o de causar uma deformação por cisalhamento nos corpos em contato.

Se não houver deslizamento entre os corpos em contato como um todo, deve haver um ponto na interface onde as superfícies se deformam sem que haja movimento relativo, o que não quer dizer que não haja deslizamento em qualquer lugar dentro da área de contato.

$\mathrm{Na}$ verdade, pode-se demonstrar que, para $\mathrm{Q}<\mu P$, ocorre um pequeno movimento relativo, isto é, um microdeslizamento na interface do contato e essas 
regiões são denominadas "slip". A outra parte, no restante da interface de contato, deforma sem movimento relativo e, nas regiões em que ocorre essa adesão, as superfícies são ditas "stick".

Dentro da região de adesão, a resultante tangencial não pode exceder seu valor limite. Assumindo-se que o coeficiente de atrito $\mu$ é constante, tem-se a seguinte relação, pela Lei de Amonton:

$$
|q(x, y)| \leq \mu \cdot|p(x, y)|
$$

Na região de deslizamento, $|q(x, y)|=\mu .|p(x, y)|$, onde $q$ deve-se opor ao sentido do deslizamento.

Como não se conhecem, a princípio, as dimensões das regiões de aderência e de deslizamento, dificuldades para a solução deste problema podem surgir. A alternativa, então, é a da tentativa e erro. Nessas circunstâncias, o primeiro passo é assumir que não há deslizamento em nenhuma parte da área de contato, para que se encontre o valor limite da força tangencial. A região onde ocorrerá o deslizamento será aquela em que a força tangencial exceder esse valor limite previamente determinado.

\subsection{DESLIZAMENTO PARCIAL ENTRE CORPOS ELÁSTICOS CILÍNDRICOS}

O método de solução deste problema foi apresentado primeiro por Cattaneo (1938) e também por Mindlin (1949).

Se a força tangencial aumenta até o valor limite de $\mu . P$, então os corpos estão a ponto de deslizarem e a tensão tangencial é dada por:

$$
\left(\mathrm{q}^{\prime} \mathrm{x}\right)=\mu p_{0}\left(1-\frac{x^{2}}{a^{2}}\right)^{\frac{1}{2}},
$$

onde $\mathrm{p}_{0}=2 \mathrm{P} / \pi \mathrm{a}$. E, além disso, é definida uma distribuição adicional de tração dada por:

$$
q^{\prime \prime}(x)=-\frac{c}{a} \mu p_{0}\left(1-\frac{x^{2}}{c^{2}}\right)^{\frac{1}{2}},
$$

que atua na região $-\mathrm{c} \leq \mathrm{x} \leq \mathrm{c}(c<a)$, conforme Figura 2.4 (JOHNSON, 1987). 


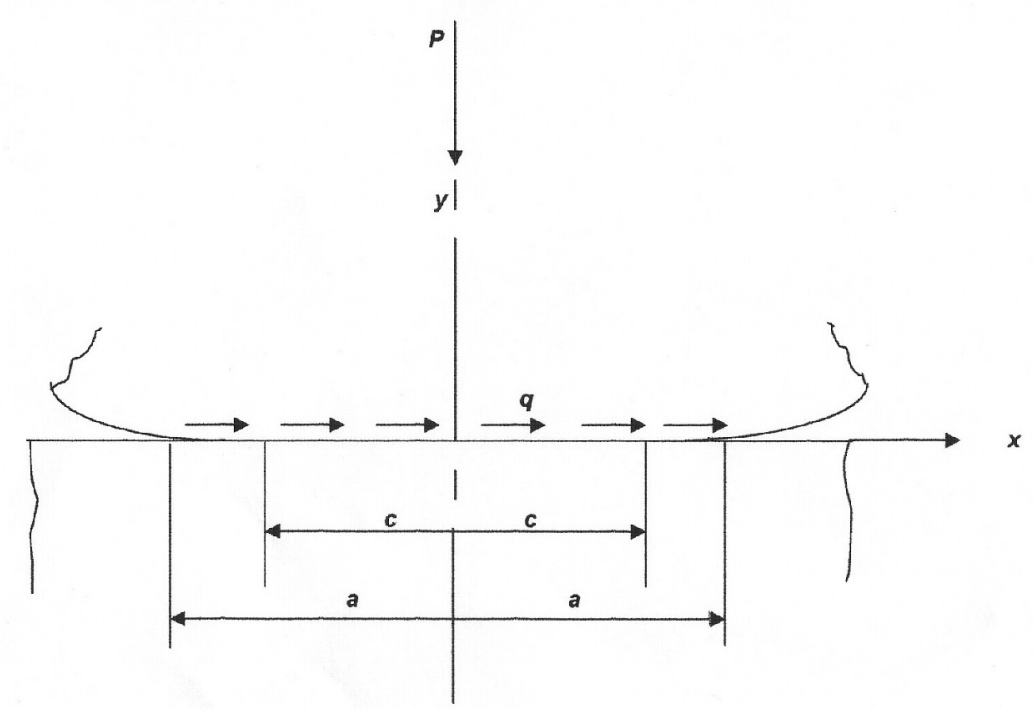

Figura 2.4 Contato entre um cilindro e um plano sujeitos a carregamentos normal e tangencial.

A tração resultante nessa região é dada por:

$$
\mathrm{q}(\mathrm{x})=\left(\mathrm{q}^{\prime} \mathrm{x}\right)+q^{\prime \prime}(x),
$$

nesse caso, em qualquer ponto, menor que $\mu \mathrm{P}$. Assim, a condição para que a região central seja uma região de adesão está satisfeita. Nas arestas de contato,

$$
\mathrm{q}(\mathrm{x})=\mu \mathrm{p}(\mathrm{x}),
$$

já que deve ser em uma região de deslizamento.

O tamanho da região de adesão é determinado pela magnitude da força tangencial,

$$
\begin{gathered}
Q=\int_{-a}^{a} q(x) d x=\int_{-a}^{a} q^{\prime}(x) d x+\int_{-c}^{c} q^{\prime \prime}(x) d x=\mu P-\frac{c^{2}}{a^{2}} \mu P \Rightarrow \\
\frac{c}{a}==\left(1-\frac{Q}{\mu P}\right)^{\frac{1}{2}}
\end{gathered}
$$

O comportamento físico, a partir da expressão (2.20), torna-se claro. Mantendo-se $P$ constante e aumentando-se $Q$ desde zero, microdeslizamentos iniciam-se imediatamente, do contorno das áreas de contato para o centro, segundo a expressão (2.21). Quando $Q$ se aproxima de $\mu \mathrm{P}, c$ se aproxima de zero e a região 
de adesão se reduz a uma linha em $\mathrm{x}=0$. Qualquer acréscimo a $Q$ que exceda $\mu \mathrm{P}$ faz com que o contato deslize.

\subsection{SOLUÇÃO ANALÍTICO-NUMÉRICA DO PROBLEMA DE CONTATO ENTRE UM CILINDRO E UM PLANO REVESTIDO}

A solução analítico-numérica para o problema em estudo, conforme Figura 2.1, foi obtida por Oliveira e Bower (1996). Nessa solução, com o objetivo de facilitar a análise, algumas hipóteses simplificadoras são adotadas na construção do modelo proposto. Uma delas é a de assumir que a distribuição de pressão, $\mathrm{p}(\mathrm{x})$, não é afetada pelos esforços tangenciais que atuam entre o cilindro e o plano semi-infinito, composto por revestimento e substrato. O primeiro passo no sentido de se resolver o problema de contato, então, é calcular os deslocamentos e o campo de tensões no semiespaço revestido, devido às trações normal e tangencial, respectivamente, $\mathrm{p}(\mathrm{x})$ e $\mathrm{q}(\mathrm{x})$, atuando na sua superfície. Esse problema específico foi resolvido por Gupta (1973).

Os deslocamentos e o campo de tensões devem satisfazer às seguintes condições de contorno, em $\mathrm{y}=0$ e para $-\mathrm{a}<\mathrm{x}<\mathrm{a}$ :

$$
\begin{gathered}
u_{y}(x)=\Delta-x^{2} / 2 R, \\
\sigma_{y}(x)<0, \sigma_{x y}(x)=\mu \cdot \sigma_{y}(x),
\end{gathered}
$$

e para $-a>x>a:$

$$
\begin{aligned}
& \mathrm{u}_{\mathrm{y}}(\mathrm{x})<\Delta-\mathrm{x}^{2} / 2 \mathrm{R} \\
& \sigma_{\mathrm{xy}}(\mathrm{x})=\sigma_{\mathrm{y}}(\mathrm{x})=0
\end{aligned}
$$

, onde $\Delta$ é a profundidade de penetração do indentador no semiplano infinito.

Os resultados, então, para as condições de contorno acima são expressos em termos de uma função de tensão de Airy, a qual deve satisfazer à equação bi-harmônica (2.24):

$$
\nabla^{4} \psi=0
$$

Disso resultam as seguintes equações, em notação indicial: 


$$
\sigma_{11}=\psi_{, 22,} \quad \sigma_{22}=\psi_{, 11,} \quad \sigma_{12}=\psi_{, 12,}
$$

$\mathrm{O}$ fato de tanto os deslocamentos $\left(\mathrm{u}_{\mathrm{x}}, \mathrm{u}_{\mathrm{y}}\right)$ quanto as trações deverem ser contínuos na interface do revestimento com o substrato, em $y=h$, conduz a um sistema de seis equações, em termos da transformada de Fourier da distribuição de tração na superfície de contato. Uma vez resolvida a transformada de Fourier, o campo de tensões é obtido das equações (2.22) e (2.23).

Vários cálculos numéricos rigorosos feitos por Gupta e Walowit (1972); King e O'Sullivan (1987); Leroy e Villechaise (1990) mostram que, na prática, a distribuição de pressão $\mathrm{p}(\mathrm{x})$ fica muito próxima à distribuição de pressão proposta por Hertz, isto é:

$$
\mathrm{p}(\mathrm{x}) \cong \mathrm{P}_{0}\left[1-\left(\frac{\mathrm{x}}{\mathrm{a}}\right)^{2}\right]^{\frac{1}{2}}
$$

O principal efeito da combinação das propriedades elásticas do revestimento com o substrato é alterar a largura de contato, $a$, e a máxima pressão de contato, $\mathrm{P}_{0}$. Portanto, para se resolver o problema de contato, é suficiente que se determinem $a$ e $\mathrm{P}_{0}$, de tal forma que satisfaçam as condições de contorno tão proximamente quanto possível da realidade. Em geral, esses resultados devem ser obtidos mediante os dados disponíveis, que, via de regra, são o raio do indentador, $\mathrm{R}$, a carga por unidade de comprimento aplicada ao indentador, $\mathrm{P}$, e as propriedades do revestimento e do substrato.

Os erros obtidos nos cálculos para todos os casos estudados por método analítico foram inferiores a $0,7 \%$, processados em FORTRAN ${ }^{\circledR} 6.0$, cujo código foi gentilmente cedido pelos autores para ser utilizado como um dos procedimentos de validação do modelo deste trabalho, que se utiliza do Método dos Elementos Finitos (MEF). 
